\title{
Hybrid Bacteria Foraging Optimization with Cuckoo Search Algorithm for Optimal Cluster head Selection in Wireless Sensor Networks
}

\author{
B. Pitchaimanickam, G. Murugaboopathi
}

\begin{abstract}
Wireless Sensor Networks (WSN) are operated on a battery source. The sensor nodes are required more energy for the communication and information processing and also reduced the network lifetime. An efficient clustering scheme is designed to reduce the energy utilization and prolonged the network lifetime. In this paper, a hybrid approach of Bacteria Foraging Optimization with Cuckoo Search algorithm is proposed in the LEACH-C algorithm. This proposed methodology improves the chemotaxis behavior of Bacteria Foraging Optimization by using the Cuckoo Search algorithm. The residual energy of the node and the minimum distance among the nodes are considered for selecting the optimal cluster head. The proposed methodology has been done in Network Simulator (NS-2) and the experimental results improve the network lifetime by increasing the alive nodes and minimize the energy consumption. The proposed methodology has provided 700 seconds of life duration to the nodes, which is significantly higher than the lifetime provided by any other conventional techniques.
\end{abstract}

Keywords: Bacteria Foraging Optimization, Cuckoo Search algorithm, Network lifetime, Wireless Sensor Networks.

\section{INTRODUCTION}

$\mathrm{W}$ ireless sensor networks have become more popular for increasing the demand in the recent years to the advance development of wireless communication and electronics [1]. These networks are mainly used for military and civil applications and also extended for the various applications such as industrial applications, precision agriculture, animal tracking, and habitat monitoring. Each sensor node is battery oriented device and it has limited amount of energy used for the processing. Adequate energy is required to transfer the information from the sensor nodes to the base station through the cluster head. The battery recharge or replacement of the sensor nodes is complicated in remote areas. Energy efficiency is one among the several issues that is required for designing the sensor networks. energy utilization and prolonged the network lifetime. The clusters are formed by collecting the group of nodes and every cluster is controlled by the cluster head, which coordinates the data transmission activities of the clusters and sends the

Revised Manuscript Received on December 16, 2019.

* Correspondence Author

B.Pitchaimanickam, Department of Computer Science and Engineering, Kalasalingam Academy of Research and Education, Tamilnadu, India. Email: bpitmani@gmail.com

G.Murugaboopathi*, Department of Computer Science and Engineering, Kalasalingam Academy of Research and Education, Tamilnadu, India. Email: gmurugaboopathi@gmail.com
A hierarchical clustering approach is utilized to overcome the

information to the base station. LEACH [2] is the well-known clustering hierarchy algorithm in wireless sensor networks. This algorithm has the following limitations: The node position and remaining energy of the nodes are not considered in the cluster head selection. The base station does not have the global knowledge of the network. The functioning of LEACH was improved by the introduction of the LEACH-C algorithm which selects the cluster head that has the higher energy level. The simulated annealing optimization is utilized to form the optimal clusters. This optimization method executes slowly for the repeated annealing schedule and consumes more energy for computing the cost function. This method affects the lifetime of the network.

Biologically inspired methods are utilized to design the sensor networks, and they provide the features of scalability, robustness and adaptation [10], [13]. The complex network system is simplified and converted into a set of rules, which has better compatibility with a new environment. Some of the biologically inspired computational methods can be utilized for solving the routing and clustering problems [11], [12].

Bacteria Foraging Optimization [14], [15] is a well known biologically inspired approach, which initiates the foraging behavior of Escherichia Coli bacteria. Self-adaptability of the individual bacteria is supported for the group searching activities, which results in the function of the optimal bacterial colony. In the bacteria foraging optimization, step size of the chemotaxis process is taken as fixed value. This process does not generate the optimal solution in the global best level. The step size of the chemotaxis process can be improved by utilizing the cuckoo search algorithm. This methodology explores to find the accurate fitness value and also to improve the global search behavior.

The main goal of this paper is to implement the hybrid approach of bacteria foraging optimization with cuckoo search algorithm in LEACH-C algorithm to minimize the energy utilization and prolong network lifetime. The proposed methodology finds the optimal cluster head by considering the residual energy and minimum distance among the sensor nodes and cluster head. The optimal cluster head is identified among the clusters that are used for saving the energy of the nodes.

The major contributions of this paper can be summarized as follows:

1) The optimal clustering problem is formulated for prolonging the network lifetime and also minimizing the energy consumption.

\section{Published By:}


2) We use a Hybrid Bacteria Foraging Optimization with Cuckoo Search algorithm (HBFOCS) to find the optimal cluster head selection by considering the remaining energy and distance between the cluster head and cluster members in the Wireless Sensor Networks.

3) The performance of the proposed methodology is evaluated through simulation studies, and also achieves the better results when compared to LEACH-C and BFO algorithms in terms of network lifetime, energy consumption and number of alive nodes in the network.

The rest of the paper is structured as follows: Section 2 describes the related works. Section 3 discusses the clustering in wireless sensor networks. Section 4 elaborates a hybrid approach of bacteria foraging optimization with cuckoo search algorithm in LEACH-C algorithm to find the optimal cluster head selection. Section 5 explicates the performance analysis of LEACH-C and BFO. Section 6 reveals the concluding remarks and the scope for future work.

\section{LITERATURE SURVEY}

Kuila and Jana [4] have presented the particle swarm optimization to perform the energy efficient routing and clustering for prolonging the network lifetime. The encoding of the particle and multi-objective function developed for routing and energy conservation of the node by using the load balancing for clustering process. Logambigai and Kannan [5] have developed a fuzzy logic based unequal clustering algorithm to form the cluster by considering the node degree and remaining energy of the cluster head for extending the network lifetime. Azharuddin, Kuila and Jana [6] have presented the distributed fault-tolerant clustering and routing algorithm, which forms the clusters by using remaining energy and distance from cluster head to nodes. During the sudden failure of the cluster head, this algorithm is utilized to recover the sensor nodes.

Baranidharan and Santhi [7] have developed a fuzzy logic based distributed unequal clustering for balancing the energy between the nodes and extended the network lifetime. The selected cluster head uses multi-hop data transmissions for reducing the energy consumption. Malathi, Gnanamurthy and Chandrasekaran [8] have proposed the hybrid unequal clustering with layering algorithm for achieving the cluster formation and prolonging the network lifetime. This algorithm is mainly utilized for dividing the network into layers and size of the clusters. The cluster formation is considered by using the energy and distance between sink and neighbor nodes. Gu, Yu, Yu, Wang and Lv [9] have developed coverage and energy aware distributed clustering protocol to consider the coverage requirements for electing the cluster head. This algorithm guarantees for improving the network coverage performance and network lifetime.

Elazim and Ali [18] have presented the cuckoo search algorithm for designing the optimal power system stabilizer. This approach presents better results than GA and conventional based method with considering the time domain and Eigen values. Goyal and Patterh [19] have presented the cuckoo search algorithm for estimating the position of the sensor nodes. This algorithm performs well when compared to PSO and biogeography based optimization with localization error. Cui, Sun, Wang, Xue and Chen [20] have designed the cuckoo search algorithm with random distribution for locating the node information in the global search behavior. Mohanty and Parhi [21] have developed the cuckoo search algorithm to find the optimal path planning for autonomous vehicles in the unknown environment.

Feng, Wang and Gao [22] have developed the hybrid approach of cuckoo search with harmony search to find the solution for 0-1 knapsack problem. This hybrid approach compared with other conventional algorithms along with the speed of convergence and search accuracy. Gupta and Jha [23] have designed an improved cuckoo search algorithm for performing the cluster head distribution and improved harmony search utilized to transmit the data between the cluster heads and sink node. This hybrid approach evaluated by considers the metrics of average energy consumption, alive nodes and network lifetime. Long, Liang, Huang and Chen [24] have proposed the hybrid cuckoo search with solis and wets local search method to perform the global optimization by using the augmented lagrangian function.

With the literature review made on a vast scale, it is evident and concrete that the utilization of Cuckoo Search algorithm with $\mathrm{BFO}$ in a LEACH-C algorithm for optimizing the position of sensor nodes is unavailable. This has obviously motivated the authors to implement and use the same to find the optimal cluster head in a wireless sensor for the better functioning of a network.

\section{CLUSTERING IN WIRELESS SENSOR NETWORKS}

Clustering is an important methodology to group the nodes together and collect the information from the nodes. The network is divided into set of clusters and the cluster head is nominated for each cluster. The cluster head coordinates the aggregation and transmission of data to the base station.

LEACH-C (Centralized) algorithm [3] was used as a central control method to generate the optimal clusters, which is utilized to identify the cluster head for the network.

Simulated annealing optimization is utilized in LEACH-C. These approaches have certain limitations. It spends more energy for forming the optimal clustering, and the sensor nodes lose energy quickly, resulting in reduced life time of the network. The above limitations are resolved by introducing hybrid approach bacteria foraging optimization with cuckoo search algorithm for reducing the energy consumption and extending the network lifetime.

\section{HYBRID BACTERIA FORAGING OPTIMIZATION WITH CUCKOO SEARCH ALGORITHM}

In the tumbling process of BFO algorithm, the step size is considered in the random direction represented by $C(i)$. The step size is modified by utilizing the cuckoo search algorithm with the Levy flight feature [16], [17]. With the help of Levy flight features for generating the efficient solution in the random walk. The bacteria will make a movement to the new position obtained by the Levy flight and this can be represented as 


$$
C(i)=C(i)+\alpha \cdot * \operatorname{Levy}(\lambda)
$$

Where $\alpha>0$ used to relate the step size of the problem. The value of $\alpha$ is used as 1 in most of the cases. The random steps are obtained by using the levy distribution, Levy $\sim u=i^{-\lambda}$ $1<\lambda \leq 3$ that has an infinite variance with infinite mean. The random walk uses the power law step length distribution. BFO algorithm uses the cuckoo search algorithm with levy flight feature for generating the better value in the evaluation of the fitness function.

Let $P_{i}(j, k, l)$ indicates the position of each $i^{t h}$ bacteria at the $j^{\text {th }}$ chemotactic, $k^{\text {th }}$ reproduction, $l^{\text {th }}$ elimination and dispersal, and $P_{i}(j, k, l)$ is represented by

$P_{i}(j, k, l)=\left\{\theta^{i}(j, k, l) / i=1,2 . . S\right\}$

$J(i, j, k, l)$ denotes the fitness function of the $i^{\text {th }}$ bacterium. The tumble step is represented as

$$
P_{i}(j+1, k, l)=\theta^{i}(j, k, l)+C(i) \varphi(j)
$$

Where, $C(i)$ is the step size and $\varphi(j)$ is the run length vector. The fitness function $J(i, j+1, k, l)$ is better than $J(i, j, k, l)$, the swimming process is to obtain the minimum value and also to repeat the process for the remaining bacteria.

The swarming process sends the signal to the cell based on the attractant and repellent method to generate the swarm patterns. The cell to cell attractant of the $i^{\text {th }}$ bacteria is represented as:

$$
J_{c c}(\theta)=\sum_{i=1}^{s} J_{c c}^{i}
$$

$=\sum_{i=1}^{s}\left[-d_{\text {attract }} \exp \left(-w_{\text {attract }} \sum_{m=1}^{p}\left(\theta_{m}-\theta_{m}^{i}\right)^{2}\right)\right]+$ $\sum_{i=1}^{S}\left[-h_{\text {repellent }} \exp \left(-w_{\text {repellent }} \sum_{m=1}^{p}\left(\theta_{m}-\right.\right.\right.$ $\theta m i) 2$

Where, $d_{\text {attract }}$ is the depth of the attractant, $w_{\text {attract }}$ is the width of the attractant, $h_{\text {repellent }}$ is the height of the repellent and $w_{\text {repellent }}$ is the width of the repellent. The main aim is to compute the least value of $J(i, j, k, l)+J_{c c}\left(\theta^{i}(j, k, l)\right)$.

After the execution of chemotaxis process, the reproduction process is executed. In the reproduction step, the population of the bacteria is divided into two bacteria. The least healthy bacterium expires from the environment and most healthy bacterium divides into two bacteria. These bacteria are located in the similar position. The elimination and dispersal process is executed after the reproduction step. Each bacterium can be moved to search the solution in the other domain.

\section{A. Optimal cluster head selection using Hybrid BFO with CS algorithm}

The algorithmic steps for the selection of optimal cluster head by using hybrid approach of Bacteria Foraging Optimization with Cuckoo Search algorithm, is shown in below:

Step 1: Assign the number of bacteria to all possible cluster head candidates and initialize the other parameters used for the Bacteria Foraging Optimization and Cuckoo Search Algorithm.

Step 2: Initialize the energy for every node and to find the minimum distance from the cluster head to base station.

Step 3: Apply the hybrid approach of Bacteria Foraging Optimization with Cuckoo Search algorithm.

Step 3.1: Apply the Levy flight method to the step size of chemotaxis process.

Step 3.2: Execute the processes involved for the bacteria foraging.

Step 3.3: Check the energy and distance of each node.

Step 3.4 If the better value is achieved in the new step then, and then consider the new cluster head in the next iteration.

Step 4: Repeat the Step3 for reaching the maximum iterations.

Step 5: Fitness value is calculated for the set of cluster heads.

Step 6: Identify the optimal cluster head with minimum fitness value.

Step 7: Optimal cluster head is informed to the same group of cluster members.

\section{B. Pseudo Code:}

Step 1: Initialize the parameters used for BFA and Cuckoo Search algorithm.

Step 2: For each elimination-dispersal loop $l: l=l+1$.

Step 3: For each reproduction loop $k: k=k+1$.

Step 4: For each chemotaxis loop $j: j=j+1$.

a) For each $i^{\text {th }}$ bacterium 1 to $S$

b) Evaluate the cost function of current position $J(i, j, k, l)$ and saved as $J_{\text {last }}$

c) Tumble: Generate the random vector $\Delta(i)$ in the range of $[-1,1]$

d) Move:

$$
\begin{aligned}
C(i) & =C(i)+\alpha \cdot * \operatorname{Levy}(\lambda) \\
J(i, j+1, k, l) & =J(i, j, k, l)+C(i) * \frac{\Delta(i)}{\sqrt{\Delta(i)^{T} \cdot \Delta(i)}}
\end{aligned}
$$

e) Swim: Let $m=0$

While $m<N_{s}$

$$
\begin{aligned}
& m=m+1 \\
& \text { if } J(i, j+1, k, l)<J_{\text {last }} \text { then } \\
& J_{\text {last }}=J(i, j+1, k, l) \\
& \text { end if }
\end{aligned}
$$

$$
J(i, j+1, k, l)=J(i, j+1, k, l)+C(i) * \frac{\Delta(i)}{\sqrt{\Delta(i)^{T} \cdot \Delta(i)}}
$$

else

$$
\begin{aligned}
& J(i, j+1, k, l)=\quad m=N_{s} \\
& J(i, j+1, k, l)+C(i) * \\
& \frac{\Delta(i)}{\sqrt{\Delta(i)^{T} \cdot \Delta(i)}}
\end{aligned}
$$

Published By: 
Repeat the steps (a) to (e) for all the chemotaxis steps.

Step 5: Reproduction: Find the value of $J_{\text {health }}$. The bacteria can be sorted according to the ascending order of $J_{\text {health }}$ and the least health value of the bacteria die and most health value of bacteria splitted into copies and placed in to the same location.

$$
\begin{aligned}
\text { a) if } J< & N_{r e} \text { then } \\
& \text { go to step } 3 \\
\text { else } & \\
& \text { go to step } 6
\end{aligned}
$$

Step 6: Elimination and dispersal:

$$
\text { for } i=1,2, \ldots S
$$

Generate the random number $r n$

if $r n \leq P_{e d}$ then

bacterium is dispersed into new random location. else end for

\section{Step 7:}

If $l<N_{e d}$ then

go to step 2

else

find the positions of the cluster head candidate. end if

\section{Computing the Optimal Clusterhead using HBFOCS}

The main aim is to compute the optimal cluster head by evaluating the fitness function. The fitness value is calculated for each cluster head. The minimum value of the fitness function identifies the optimal cluster head.

$$
F=f 1 * \alpha+f 2 *(1-\alpha)
$$

Where,

$f 1=\frac{\sum_{i=1}^{N} E\left(n_{i}\right)}{\sum_{i=1}^{N} E\left(C_{i}\right)}$

$f 2=\max \sum_{i \in j} d\left(n_{i}, C H_{j}\right) /\left|C_{j}\right|$

The fitness value is computed by utilizing the remaining energy and minimum distance between the nodes and cluster head. Equation.7 denotes the sum of energy consumed for each sensor nodes divided by the sum of energy required by the cluster head. Equation. 8 represents the highest average distance between the cluster head and the nodes divided by the number of nodes available in the cluster. The optimal cluster head ensures the minimum distance and higher residual energy. The parameters of the HBFOCS algorithm are shown in Table. 1

\section{Table- I: Parameters of HBFOCS}

\begin{tabular}{|l|l|}
\hline Parameters & Value \\
\hline Population of bacteria & 100 \\
\hline Chemotactic steps & 100 \\
\hline Swim length of bacteria & 4 \\
\hline Reproduction steps & 4 \\
\hline Elimination and dispersal steps & 2 \\
\hline Probability of elimination dispersal & 0.25 \\
\hline Width of attractant & 0.04 \\
\hline Depth of attractant & 0.01 \\
\hline Height of repellent & 0.01 \\
\hline Width of repellent & 10 \\
\hline
\end{tabular}

\section{A. Simulation Model}

The sensor nodes are randomly deployed in the topological area and the nodes have used the same transmitting and receiving capabilities. The equal energy is assigned to all the sensor nodes at the initial period. The simulation is performed by using NS2-27. In these experiments, the sensor nodes are deployed in $100 \times 100 \mathrm{~m}^{2}$. The base station is placed at the position of $(50,175)$ and the initial energy of the node is considered between $1 \mathrm{~J}$ and $5 \mathrm{~J}$. The percentage of cluster head nodes are considered as $5 \%$ of the number of nodes in each round. The simulation time of each experiment is 3600 seconds.

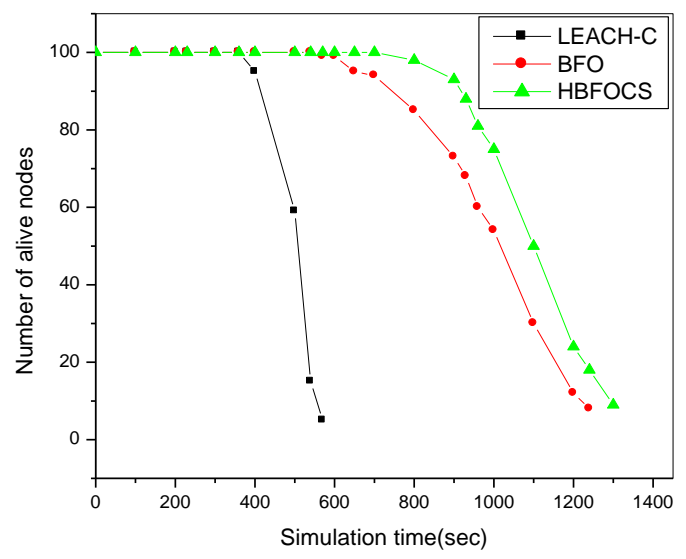

Fig.1 Effect of alive nodes on simulation time

Figure 1 shows an effect of alive nodes with simulation time for LEACH-C, BFO and HBFOCS algorithms. The first node dead value of LEACH-C, BFO and HBFOCS algorithms is 360,540 and 700 seconds respectively. The proposed methodology increases the alive nodes when compared to LEACH-C and BFO algorithms.

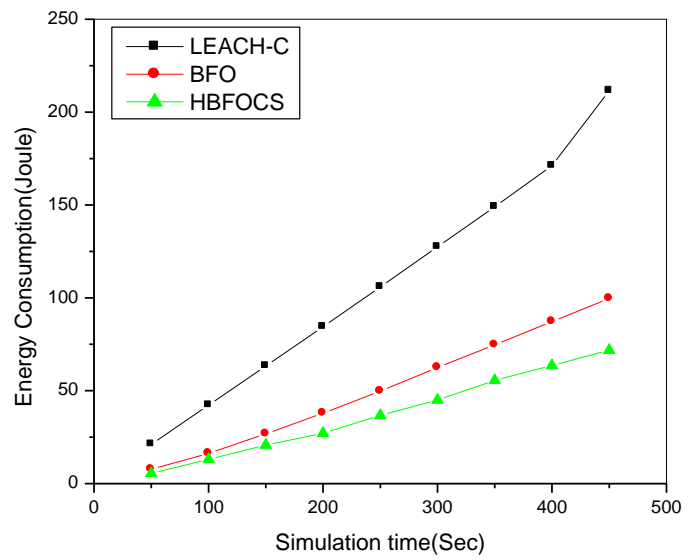

Fig.2 Effect of energy consumption

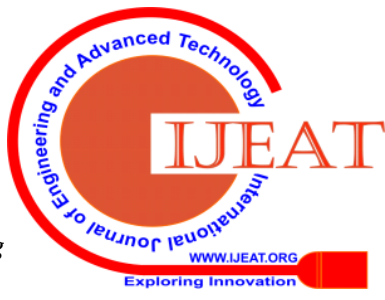


Figure 2 illustrates an effect of consuming the energy along with simulation time. Energy efficiency is the significant factor for prolonging the network lifetime. The proposed methodology consumes minimum amount of energy for the communication process when compared to LEACH-C and $\mathrm{BFO}$ algorithms. The performance of BFO is improved with the help of cuckoo search algorithm which identifies the optimal cluster head from the set of clusters.

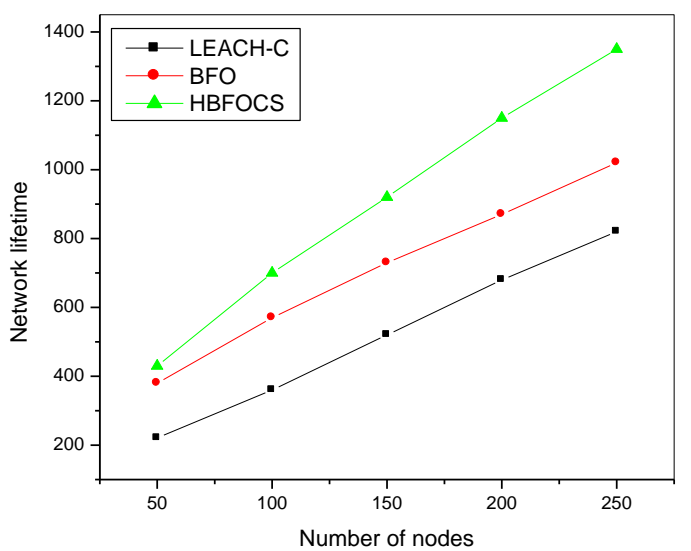

Fig.3 Effect of network lifetime (FND) under increasing nodes

Figure 3 demonstrates an effect of the network lifetime by increasing the sensor nodes for different clustering algorithms. The network lifetime is calculated by using the first node dead. The proposed methodology presents the better network lifetime when compared to LEACH-C and BFO algorithms.

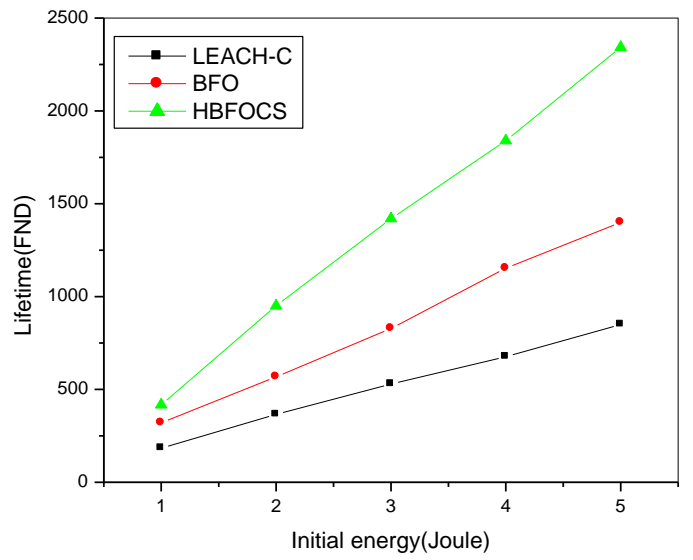

Fig.4 Effect of Network Lifetime (FND) Vs Initial Energy

Figure 4 shows an effect of network lifetime by utilizing the different initial energy level of the nodes from $1 \mathrm{~J}$ to $5 \mathrm{~J}$. The proposed methodology achieves the better performance in lifetime when compared to the LEACH-C and BFO algorithms. The functionality of the cuckoo search algorithm is included in BFO for improving the network performance which provides the better network lifetime and minimum energy consumption.

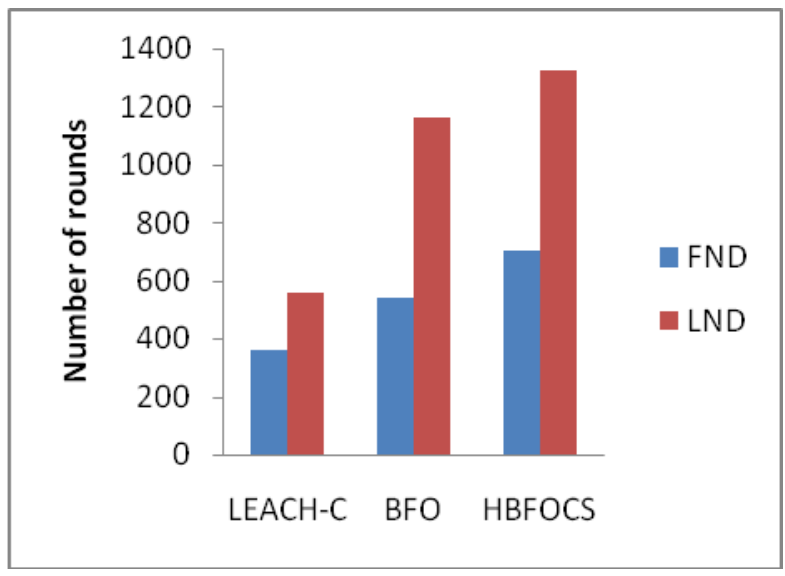

Fig.5 Comparison of network lifetime with FND and LND value - base station outside the region $(50,175)$

Figure 5 illustrates an evaluation of the network lifetime by considering the outside of the base station with the values of FND and LND. The rounds of FND and LND are measured for the LEACH-C, BFO and HBFOCS algorithms. The proposed methodology gives the better performance for network lifetime when compared to the LEACH-C and BFO algorithms.

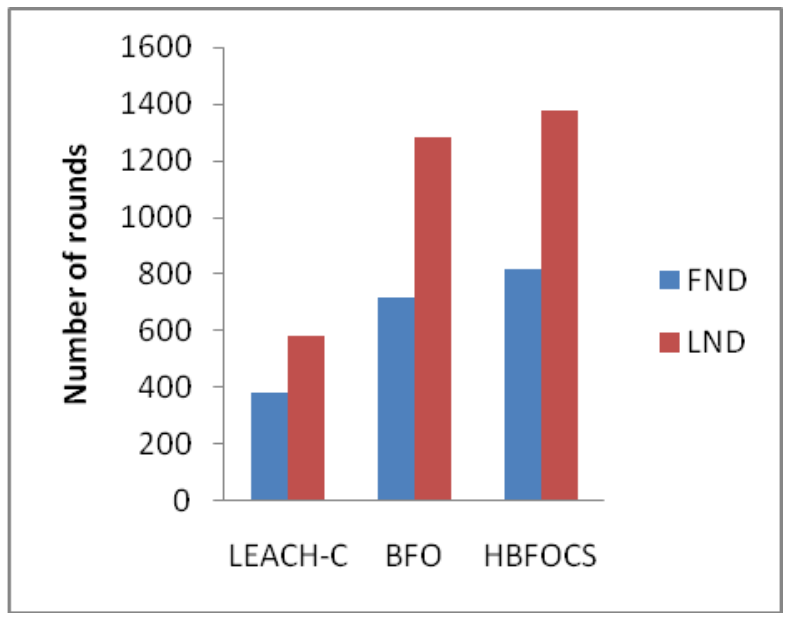

Fig. 6 Comparison of Network lifetime with FND and LND base station inside the region $(50,50)$

Figure 6 shows the measure of network lifetime by considering the base station available at the position of $(50$, 50). The proposed methodology maintains all the nodes are alive upto 820 seconds and also increases the alive nodes for long period of time. The proposed methodology achieves the better results when compared to the LEACH-C and BFO algorithms.

The approximated pareto front analysis is done by measuring the network lifetime with different positions of base station. The FND dead occur at 820 second for using the base station available inside the region. The nodes are alive upto 700 seconds for using the base station outside of the region. 


\section{CONCLUSION}

Clustering is an important method for providing energy efficiency in the wireless sensor networks. The hybrid approach of Bacteria Foraging Optimization with Cuckoo search algorithm is proposed in LEACH-C algorithm for optimizing the cluster head selection process with considering the remaining energy and the minimum distance among cluster head and the sensor nodes. The results should yield an optimal governance of energy that is needed for prolonging lifetime of the network. The proposed methodology delivers better results for the network lifetime and energy consumption when compared to the LEACH-C and BFO algorithms.

The experimental results illustrate that the proposed methodology minimizes the energy consumption and also extends the lifetime of the network by increasing the number of alive nodes. This paper provides a possible scope for future with two main aspects: 1) A hybrid approach that combines the other biologically inspired algorithms with BFO could be developed for the improvement of the lifetime. 2) The multi-objective BFO could be utilized to estimate the optimal clusters for extending the network lifetime.

\section{ACKNOWLEDGMENT}

The authors would like to thank Kalasalingam Academy of Research and Education for supporting this work.

\section{REFERENCES}

1. Akyildiz I.F, Su W, Sankarasubramaniam Y, Cayirci E, "Wireless sensor networks: a survey", Computer Networks, vol.38, no.4, pp.393-422, 2002.

2. Heinzelman W.R, Chandrakasan A, Balakrishnan H, "Energy-efficient communication protocol for wireless micro sensor networks", Proc. of $33^{\text {rd }}$ Hawaii International Conference on System Science, IEEE, 2000.

3. Heinzelman W.B, Chandrakasan A.P, Balakrishnan H, "An application-specific Protocol architecture for wireless micro sensor networks", IEEE Transaction on Wireless Communications, vol. 1, no.4, pp. 660-670, 2002.

4. Kuila P, Jana P.K, "Energy efficient clustering and routing algorithms for wireless sensor networks: Particle Swarm Optimization approach", Engineering Applications of Artificial Intelligence, vol.33, pp.127-140, 2014.

5. Logambigai R, Kannan A, "Fuzzy logic based unequal clustering for wireless sensor networks", Wireless Networks, vol.22, no.3, pp.945-957, 2016.

6. Azharuddin M, Kuila P, Jana P.K, "Energy Efficient fault tolerant clustering and routing algorithms for wireless sensor networks", Computers and Electrical Engineering,vol.41, pp.177-190, 2015.

7. Baranidharan B, Santhi B, "DUCF: Distributed load balancing unequal clustering in wireless sensor networks using Fuzzy approach", Applied Soft Computing, vol. 40, pp.495-506, 2016.

8. Malathi L, Gnanamurthy R.K, Chandrasekaran K, "Energy efficient data collection through hybrid unequal clustering for wireless sensor networks", Computers and Electrical Engineering, vol.48, pp.358-370, 2015.

9. Gu X, Yu J, Yu D, Wang G, Lv Y, "ECDC: An Energy and coverage aware distributed clustering protocol for wireless sensor networks", Computers and Electrical Engineering, vol.40, no.2, pp.384-398, 2014.

10. Ren H, Meng M.Q.H, "Biologically Inspired Approaches for Wireless Sensor Networks", Proc. of 2006 International Conference on Mechatronics and Automation, pp.762-768, IEEE, 2006.

11. Iyengar S.S, Wu H.C, Balakrishnan N, Chang S.Y, "Biologically Inspired Cooperative Routing for Wireless Mobile Sensor Networks". IEEE Systems Journal, vol.1, no.1, pp.29-37, 2007.

12. Selvakennedy S, Sinnappan S, Shang Y, "A biologically-inspired clustering protocol for wireless sensor networks", Computer Communications, vol. 30, no.14-15, pp.2786-2801, 2007.
13. Kulkarni R.V, Venayagamoorthy G.K, "Bio-inspired Algorithms for Autonomous Deployment and Localization of Sensor Nodes", IEEE Transaction on System, Man and Cybernetics Part C: Applications and Reviews, vol. 40, no.6, pp.663-675, 2010.

14. Passino K.M, "Biomimicry of bacterial foraging for distributed optimization and control", IEEE Control System, vol.22, no.3, pp.52-67, 2002.

15. Liu Y, Passino K.M, "Biomimicry of Social Foraging Bacteria for Distributed Optimization: Models, Principles, and Emergent Behaviors", Journal of optimization theory and applications, vol.115, no.3, pp. 603-628, 2002.

16. Yang X.S, Deb S, "Cuckoo Search via levy flights", Proc. in2009 World Congress on Nature and Biologically Inspired Computing, pp.210-214, IEEE, 2009.

17. Yang X.S, Deb S, "Engineering Optimization by Cuckoo Search", International Journal Mathematical modeling and Numerical optimization, vol.1, no.4, pp.330-343, 2010.

18. Elazim S.A, Ali E.S, "Optimal power system stabilizers design via cuckoo search algorithm”, International Journal of Electrical power and Energy Systems, vol.75, pp.99-107, 2016.

19. Goyal S, Patterh M.S, "Wireless Sensor Network localization based on Cuckoo Search algorithm", Wireless Personal Communications, vol.79, no.1, pp.223-234, 2014.

20. Cui Z, Sun B, Wang G, Xue Y, Chen J, “A novel oriented cuckoo search algorithm to improve the DV-Hop performance for cyber-physical systems", Journal of parallel and distributed computing, vol.103, pp.42-52, 2017.

21. Mohanty P.K, Parhi D.R, "Optimal path planning for a mobile robot using cuckoo search algorithm', Journal of Experimental and Theoretical Artificial Intelligence, vol. 28, no.(1-2), pp.35-52, 2014.

22. Feng Y, Wang G.G, Gao X.Z, "A Novel Hybrid Cuckoo Search Algorithm with Harmony Search for 0-1 Knapsack problems", International Journal of Computational Intelligence Systems, vol. 9, no.6, pp.1174-1190, 2016.

23. Gupta G.P, Jha S, "Integrated clustering and routing protocol for wireless sensor networks using Cuckoo and Harmony search based meta heuristic techniques", Engineering Applications of Artificial Intelligence, vol.68, pp.101-109, 2018.

24. Long W, Liang X, Huang Y, Chen Y, "An effective hybrid cuckoo search algorithm for constrained global optimization", Neural computing and applications, vol.25, no.(3-4), pp.911-926, 2014.

\section{AUTHORS PROFILE}

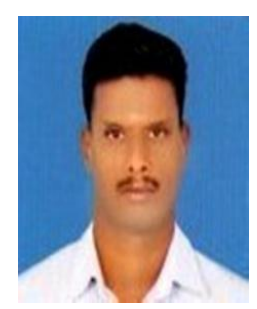

B.Pitchaimanickam received his Undergraduate degree in Computer Science and Engineering from Madurai Kamaraj University. He received a Post graduate degree in Computer Science and Engineering from Anna University. He is currently working as an Assistant professor in Kalasalingam Academy of Research and Education, Krishnankoil, Tamilnadu. His area of interest includes optimization techniques and Wireless Sensor Networks.

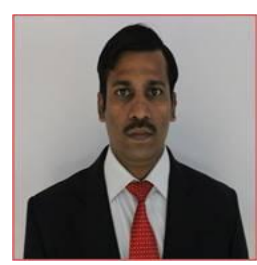

G.Murugaboopathi received the Undergraduate Degree in Computer Science and Engineering from Madurai Kamaraj University, the Post Graduate degree in Digital Communication and Network from Madurai Kamaraj University, and Ph.D in Computer Science and Engineering at Bharath University, Chennai. He has more than 35 publications in National, International Conference and International Journal proceedings. He is currently working as an Associate Professor in the Department of Computer Science and engineering at Kalasalingam Academy of Research and Education, Krishnankoil, Virudhunagar Dist, Tamilnadu, India He has more than 13 years of teaching experience. His areas of interest include Wireless Sensor Networks, Mobile Communication, Mobile Computing and Mobile Adhoc Networks. 